\title{
The Association Between Abstinence Period And Semen Parameters: Results In Normal Samples And Different Sperm Pathology
}

\section{Min Xie}

University Hospital Zurich

Silvan Haemmerli ( $\nabla$ silvan.haemmerli@gmail.com )

University Hospital Zurich

Kerstin Blickenstorfer

University Hospital Zurich

\section{Brigitte Leeners}

University Hospital Zurich

\section{Research Article}

Keywords: abstinence, Sperm, percentage, morphology

Posted Date: November 16th, 2021

DOI: https://doi.org/10.21203/rs.3.rs-576492/v2

License: (c) (i) This work is licensed under a Creative Commons Attribution 4.0 International License.

Read Full License 


\section{The association between abstinence period and semen}

2 parameters: Results in normal samples and different sperm

3 pathology

4 Min Xie ${ }^{1}$, Silvan Haemmerli (ORCID-ID 0000-0002-08753-3007) ${ }^{1 *}$, Kerstin Blickenstorfer ${ }^{1}$, Brigitte Leeners

5 (ORCID-ID 0000-0003-4027-6151) $)^{1,2}$

6 First and second authors have contributed equally to the manuscript

$7 \quad *$ Corresponding author

8

$9 \quad{ }^{1}$ University Hospital Zurich, Dept. of Reproductive Endocrinology, 8091 Zurich, Switzerland

$10 \quad{ }^{2}$ University of Zurich, 8091 Zurich, Switzerland

Short title: Abstinence period and semen parameters

Key words: abstinence period, sperm quality, sperm concentration, sperm motility, sperm morphology

Corresponding author:

Dr. med. Silvan Haemmerli

Department of Reproductive Endocrinology

Frauenklinikstr. 10 
Purpose: The length of sexual abstinence seems to influence sperm quality. However, few data on the relevance of abstinence time in pathological sperm samples are available. With our study, we look for associations of abstinence length and semen quality.

Methods: We studied semen samples from 4423 men undergoing fertility evaluation. Sperm concentration, percentage of progressively motile spermatozoa, total motile sperm count, percentage of spermatozoa with normal morphology, were compared after each day and 0-2, 3-7 and $>7$ days of abstinence.

Results: We found that a longer abstinence time was related to higher sperm concentration in normal semen samples $(\mathrm{P}<0.001)$ and in semen samples with any sperm pathology $(\mathrm{P}<0.001, \mathrm{P}=0.004)$ with the exception of oligozoospermia $(\mathrm{P}=0.125)$. Longer abstinence time was also associated with significantly reduced progressive motility in normal samples $(\mathrm{P}<0.001)$ and in cases of teratozoospermia $(\mathrm{P}<0.001)$. In normal samples a higher percentage of sperm were morphologically normal after a shorter abstinence period $(\mathrm{P}=0.03)$; in oligoasthenoteratozoospermia (OAT) samples, this was the case after a longer abstinence period $(\mathrm{P}=0.013)$.

Conclusion: A longer abstinence time is associated with higher sperm concentration, whereas sperm motility is optimal after shorter abstinence times; results on morphology are controversial. The recommendation on abstinence time needs to be adjusted in relation to the parameter that needs to be improved.

INTRODUCTION

As male factors contribute to between $30 \%$ and $50 \%$ of cases of infertility ${ }^{1,2}$, the examination of semen quality is a standard element in a male fertility assessment. Sperm quality not only influences natural conception but also influences the outcome of intrauterine inseminations (IUI) and in vitro fertilization (IVF)/ intracytoplasmic sperm injection (ICSI). Of particular importance are sperm concentration, the percentage of motile spermatozoa, and the percentage of morphologically normal spermatozoa ${ }^{3-5}$. Success rates of IUI are significantly higher when total motile sperm count (TMSC) is above $5-10$ million (m) than when TMSC is below $5 \mathrm{~m}^{5}$. Studies of men presenting for IUI indicate that a short abstinence leads to higher TMSC $^{6,7}$ and increased values for sperm concentration, motility, and normal morphology ${ }^{8}$. The likelihood of significant improvement in TMSC and in total normal morphology count (TNMC) in the second analysis increased in function of the number of subnormal semen parameters in the first analysis ${ }^{6}$. In contrast, most men with normal or few subnormal semen parameters showed deteriorated values in the second ejaculate. However, data on the association between abstinence time and different types of semen pathology are rather limited. In IVF, fertilization 
and pregnancy rates correlate positively with sperm motility ${ }^{3}$. Results for the significance of morphology, however, are controversial, with some studies supporting an important role in the chances for pregnancy ${ }^{3,4}$ and others reporting very limited relevance ${ }^{9-11}$.

The abstinence period prior to semen analysis or prior to providing sperm for fertility treatments currently recommended by the World Health Organization (WHO) is 2-7 days ${ }^{12}$. However, the manual gives no specific background for the selection of this exact time period. The length of the abstinence period has been reported to have an effect on sperm concentration, motility, and morphology in normozoospermic men. However, the ideal abstinence period, especially in subfertile men, is a subject of controversy, e.g. abstinence time might influence semen parameters differently in fertile and subfertile men ${ }^{13,14}$. While some studies show a positive effect of abstinence times shorter than 2 days on sperm concentration and motility in subfertile men ${ }^{8,15}$, other authors found an increased motility after an abstinence period of longer than 4 days ${ }^{16,17}$.

The magnitude of change in sperm parameter values after different abstinence periods can be considerable: in a longitudinal study on 6 normozoospermic men, sperm count and TMSC increased 1.75 fold after 5 days of abstinence compared with 1 day ${ }^{18}$. In contrast, in most men with 2 or 3 subnormal sperm parameter values, TMSC and TNMC doubled after 1 day of abstinence compared with 2-4 days ${ }^{6}$.

While such changes might be clinically irrelevant in normozoospermia, variations in abstinence time would represent a relatively convenient and cost-effective method to improve semen parameters and associated chances for naturally conceived or medically induced pregnancy in cases of abnormal semen parameters. Aside from the treatment of infection and possibly varicosis, there are currently very few options to increase semen quality ${ }^{19}$, which makes any available option even more important. However, data are currently lacking on the relevance of abstinence time for specific pathologies such as astheno- and teratozoospermia as well as oligoasthenozoospermia, i.e. in male sub- or infertility, where improvement of sperm quality is important to augment chances for pregnancy. Last but not least, lack of an association between the length of abstinence and sperm quality would call for dropping current advice to refrain from sexual activity, consequently reducing psychological burden in patients having to deal with infertility.

Therefore, the objectives of our study were (i) to evaluate whether sperm quality differed after various durations of abstinence, and (ii) to investigate how abstinence period was associated with changes in sperm parameters in men with normal semen samples compared with men with pathological parameters.

\section{MATERIALS AND METHODS}


Study design: This is a retrospective cohort study evaluating data from semen analyses from men undergoing fertility evaluation at the andrology laboratory of the Department of Reproductive Endocrinology, University Hospital Zurich, Switzerland.

4 Patients: The study includes results from the analysis of 4423 semen samples from 4423 men who underwent a first semen analysis between October 2003 and July 2015. We excluded men undergoing cryopreservation of sperm prior to chemo- and/or radiation therapy because of cancer. As some antibiotics may affect semen quality, men who took antibiotics in the 3 months prior to the study period were excluded from the analysis. For the multiple regression analysis, we excluded participants with at any missing criteria (concentration, motility, and morphology) and participants with a value of $0 \%$ for spermatozoa with normal forms $(\mathrm{n}=458)$ or for spermatozoa with normal motility ( $n=19)$. Informed consent was obtained by all participants for this study.

Semen analysis: During the study period 2 different editions of the WHO laboratory manual for the examination and processing of human semen were valid: the $4^{\text {th }}$ edition and the $5^{\text {th }}$ edition ${ }^{12}$. For the present study, we based diagnosis of all semen analyses on criteria in the $5^{\text {th }}$ edition. Semen samples were collected by masturbation at the andrology laboratory of the Department of Reproductive Endocrinology. Patients were advised by telephone and/or by a written information sheet to have no ejaculation for at least 2 days and for a maximum of 7 days prior to sample collection. When providing the sample, they received a questionnaire on confounders of semen analysis, which included a question on the number of days of abstinence prior to collecting the sample. Samples were analysed within one hour after ejaculation. After liquefaction, semen volume was measured using a graduated serological pipet or a conical tube. Sperm concentration and motility were determined by counting the spermatozoa using a validated Makler chamber. All Makler chambers used in the andrology laboratory were validated using Accu bead+ kit (Hamilton Thorne, Parallabs, U.K). Motility was graded into 4 different categories: progressive motility (A: fast and B: slow progressive motility), non-progressive motility (C), and immotile spermatozoa (D). Vitality was assessed using the eosin-nigrosin test. Leukocytes were identified by checking for peroxidase-active cells in the semen samples. For the morphology assessment, a smear from each sample was air-dried and then fixed and stained, using the Papanicolaou staining method. Sperm morphology and immature germ cells were evaluated by visually inspecting at least 200 spermatozoa per sample using bright-field microscopy. Tygerberg strict criteria ${ }^{20}$ were applied. The same team of trained laboratory technicians performed all assessments during the study period. Although cut-off values for normal parameters vary between the $4^{\text {th }}$ and $5^{\text {th }}$ editions of the WHO manual, analysis followed the criteria in the $5^{\text {th }}$ edition throughout the study period. The laboratory participates regularly in an external quality control, QuaDeGA (quality control programme of the German Society of Andrology). The semen parameters evaluated in the present study were (1) semen volume in millilitres, (2) sperm concentration in $\mathrm{m} / \mathrm{ml}$, (3) sperm motility, specifically the percentage of progressive motility, and the percentage 
of total motility (combination of progressive and non-progressive motility), and (4) total motile sperm count (calculated by multiplying the semen volume with the sperm concentration and the percentage of total motile spermatozoa for each sample). Additionally, (5) vitality, (6) percentage of spermatozoa with normal forms, (7) percentage of immature germ cells, and (8) the presence of leukocytes in $\mathrm{m} / \mathrm{ml}$ were evaluated.

Definitions: In accordance with the lower reference values in the $5^{\text {th }}$ edition of the WHO laboratory manual, samples were grouped into categories as illustrated in Table 1. Samples with values above reference limits for sperm concentration, percentage of progressively motile spermatozoa, and percentage of normal forms were considered to be "normal samples". A sample with more than one abnormal semen parameter was taken into consideration for the comparison of each "pathological" category with normal samples. Abstinence period was divided into the following intervals: 0-2 days, 3-7 days, and $>7$ days.

Statistical analysis: We evaluated abstinence time in days as a continuous variable and, to provide a basis for clearer clinical recommendations, also in different time categories, i.e. 0-2 days, 3-7 days $>7$ days.

After confirmation of adequate distribution of the sperm parameters, a Kruskal-Wallis test was conducted to determine if there were differences in the median semen parameter values among the samples with different abstinence times for different types of sperm pathology, i.e. normal, oligozoospermic, asthenozoospermic, teratozoospermic and OAT samples. If median values were statistically significantly different among the abstinence interval groups, pairwise comparisons were performed using Dunn's procedure with a Bonferroni correction for multiple comparisons. P-values $<0.05$ were regarded as statistically significant. Differences in the number of immature germ cells between the different types of sperm pathology were first evaluated independently from abstinence period with a Mann-Whitney U test and then investigated with regard to abstinence period. A multiple regression analysis was performed to evaluate associations between total abstinence time (days) and sperm concentration, total motile spermatozoa, and spermatozoa with normal morphology based on the different types of semen pathology. All statistical testing was done with SPSS Statistics, Version 24.0 (IBM Corp. released 2016. IBM SPSS Statistics for Windows, Version 24.0. Armonk, NY: IBM Corp).

The data that support the findings pf this study are available from the corresponding author upon reasonable request.

\section{RESULTS}

The mean age of the patients was 37.7 years (range 21-79 years) at the time of providing the sample. Descriptive statistics of the sperm parameters in normal and pathological semen analyses are presented in Table 2. Table 3 presents the regression analysis evaluating the association between the number of days of the abstinence prior to 
semen collection and concentration, motility, and morphology in the total study group and in men receiving positive diagnoses of semen pathology.

Table 4 show the median values of the sperm parameters in each category of abstinence time for the normal samples. Median values for oligo-, astheno-, teratozoospermic and OAT samples in different categories of abstinence times are summarized in Figure 1.

In normal semen samples a longer duration of abstinence was related to higher sperm concentration but significantly reduced progressive motility and normal morphology. Comparing results after 0-2, 3-7 and $>7$ days confirmed the association between the duration of abstinence time and sperm concentration, with peak values for TMSC after $>7$ days of abstinence but showed no effect for progressive motility and morphology. In oligozoospermia abstinence time was not associated with either concentration, motility, or morphology. However, when the categories of abstinence time were evaluated, an interval $>7$ days was negatively correlated with sperm concentration, TMSC, progressive motility, and total motility. In asthenozoospermic samples a longer abstinence time was associated with higher sperm concentration, but there were no differences in motility or morphology. The analysis of the different abstinence time categories confirmed an association of more than 7 days of abstinence with higher sperm concentration and higher TMSC, as well as the lack of any association with motility. Morphology was best in semen samples collected after 0-2 days of abstinence. In teratozoospermia associations with abstinence time were shown for concentration and motility. Concentration was highest after $>7$ days of abstinence. Abstinence time of 3-7 days and longer was associated with elevated TMSC, and motility was highest after 3-7 days of abstinence. In both analyses no association with morphology was found. In the OAT samples a longer abstinence period was associated with a higher sperm concentration and better morphology when abstinence time was evaluated as a continuous variable. Comparison of results in the 3 different abstinence time categories did not confirm any of these findings, but TMSC was significantly higher after 3-7 days and $>7$ days of abstinence. The median percentage of total motile spermatozoa was significantly higher after 3-7 days of abstinence compared with 0-2 days. Progressive motility did not show any differences among the 3 categories of abstinence time.

\section{DISCUSSION}

Overall abstinence time is associated with sperm concentration in particular, but motility and morphology also show different associations in normal and pathological semen samples. The analysis of abstinence days as a continuous variable and the comparison of results in 0-2, 3-7 and $>7$ days led to similar results in most but not in all analyses. 
Concentration, volume, and TMSC: Our results for sperm concentration, volume, and TMSC are comparable with a cross sectional analysis of semen samples from normo- and oligozoospermic men providing ejaculates after abstinence times of 0-14 days ${ }^{15}$. For the normozoospermic samples in our study, sperm concentration and TMSC peaked after 7 days of abstinence, thus on the upper end of the abstinence of 2-7 days recommended by WHO ${ }^{12}$. The evaluation of 6 consecutive sperm samples after 1-11 days of abstinence in normozoospermic men also showed the highest sperm concentrations after $>7$ days of abstinence ${ }^{18}$; smaller longitudinal studies confirmed these results ${ }^{21,22}$.In oligozoospermic samples, Levitas ${ }^{15}$ found a higher sperm concentration after 3-5 days and peak TMSC after 4 days of abstinence; these findings are consistent with our results from the evaluation of abstinence time categories, but not with our results from the analysis of days of abstinence as a continuous variable. In addition to findings from available research, our study shows also that in astheno-, teratozoospermic, and OAT samples, semen concentration increases after longer periods of abstinence. The improvement of sperm quality is obligatory for fertility in cases of semen pathology; an increase in concentration may help to compensate deficits in motility and morphology by increasing the total number of sperm. Therefore, the selection of a period of abstinence at the higher end of the time frame recommended by WHO might contribute to the optimization of semen samples for natural and medically assisted conception. The lower sperm concentrations after shorter abstinence times for normozoospermic samples could be explained by depletion of the stored spermatozoa in the cauda epididymis when ejaculation occurs more frequently ${ }^{18}$. A longer transport time through the epididymis (3 times longer in men with oligo- than with normozoospermia) likely influences sperm concentration in oligozoospermic men in relation to abstinence times ${ }^{23}$. As previous results indicate, shorter abstinence times are associated with lower semen volume in normal and subnormal semen samples $15,18,21,22$.

Motility: When evaluating the days of abstinence as a continuous variable, longer abstinence time was associated with lower motility in normal and teratozoospermic samples. We found the highest values for total motility after 0-2 and 3-7 days of abstinence in the oligo- and the teratozoospermic samples. The normal, asthenozoospermic, and OAT samples did not show any significant difference in relation to abstinence time categories. In agreement with our findings, shorter abstinence time was associated with high motility for normal and some types of abnormal samples in other studies ${ }^{15}$. However, longitudinal studies in smaller collectives did not find any association between abstinence period and changes in motility ${ }^{21,24,25}$; this supports our findings that short abstinence times are not associated with higher motility in asthenozoospermic samples, either alone or in combination with other semen pathology, i.e. where an improvement of motility would be obligatory to improve fertility.

A possible explanation for a beneficial effect of short abstinence times is the transit time of spermatozoa through the epididymis and its consequences: more frequent ejaculation may lead to shorter storage times of spermatozoa 
in the epididymis. During their stay in the epididymis, spermatozoa are exposed to reactive oxygen species (ROS), produced by the spermatozoa and to a lesser extent by leukocytes. ROS can induce lipid peroxidation of unsaturated fatty acids in the plasma membrane of spermatozoa ${ }^{26,27}$. Various authors report unusually high levels of ROS in infertile men ${ }^{26,28}$. It is suggested that overly high levels of ROS cannot be counterbalanced by antioxidants in seminal plasma (such as catalase or superoxide dismutase) and consequently lead to oxidative stress, which has a negative impact on sperm motility ${ }^{27}$. Interestingly, seminal total antioxidant capacity was significantly higher after 1 day than after 4 days of abstinence ${ }^{25}$; this result supports the benefit of shorter abstinence time with regard to sperm motility.

Yet, there are more factors that have a modulatory effect on sperm motility: a positive correlation between levels of neutral alpha-glucosidase and motility has been demonstrated ${ }^{29,30}$. Elzanaty ${ }^{29}$ reported higher levels of neutral alpha-glucosidase in semen samples produced after 4-7 days of abstinence in comparison with shorter abstinence times. Although we did not observe a clear benefit of short abstinence times in all types of sperm pathology, when an association could be demonstrated, shorter abstinence times were always related to higher motility and can therefore be recommended when higher motility is needed.

Morphology: In our study, a shorter abstinence period was associated with a higher percentage of morphologically normal spermatozoids in normal samples and in OAT samples when days of abstinence were evaluated as a continuous variable. Teratozoospermic samples showed a higher median percentage of morphologically normal spermatozoa after 0-2 days in comparison with 3-7 days of abstinence. According to our results and those of another large cohort study ${ }^{15}$, shorter abstinence periods were associated with a higher percentage of morphologically normal spermatozoids. Again, results varied strongly between different pathologies, with teratozooic samples presenting best results in the $0-2$ days abstinence window and OAT samples showing significant association with shorter abstinence time in the analysis of abstinence days as a continuous variable. A benefit of a short abstinence time was also observed in other studies investigating subnormal semen samples ${ }^{6,8}$, but there are also studies showing no association between abstinence time and changes in morphology in both normo- and oligozoospermic men ${ }^{17,25,31,32}$. In contrast, a study investigating semen samples from men who abstained sequentially for 1-10 days reported a significant increase of the normal spermatozoa after 2-5 days of abstinence and a significant decrease after 10 days ${ }^{24}$. As ROS levels correlate negatively with the percentage of spermatozoa with normal and borderline morphology ${ }^{33}$, increased exposure to ROS in the epididymis might be involved in the decreasing numbers of morphologically normal spermatozoa after prolonged abstinence periods. Strengths and limitations: Our study reports data from one of the largest groups of men in infertile relationships. In comparison with existing studies, we not only investigated the difference between normal and oligozoospermic semen samples but also included asthenozoospermic, teratozoospermic, and OAT samples. Limiting factors of our 
study are the retrospective design and the small cohorts in some of the pathological categories. As we excluded men with $0 \%$ of motile sperms or sperms with normal morphology, our findings are not representative for men with severe cryptozoospermia or asthenozoospermia. As most of the men followed the advice to remain abstinent 2-7 days prior to sample production, there were few participants with an abstinence outside of this time window.

Because of the small number of samples in certain categories, the results for OAT samples in particular should be interpreted with caution. False answers to fulfil the expectation of adherence to the recommended abstinence time likely result in underestimation of our findings. Another limitation is the cross-sectional design with only one sample per patient, as possible within-subject variability is not accounted for. Studies on this topic found that values for concentration, motility, percentage of normal forms, and sometimes volume might vary considerably between different samples of the same patient: within-subject coefficients of variation were calculated between $25-28 \%$ for ejaculate volume, $26-29 \%$ for sperm concentration, $18-34 \%$ for total motility, and $19-29 \%$ for morphology ${ }^{34-36}$. However, in all 3 studies the between-subject coefficients of variation exceeded the withinsubject coefficients of variation; intraclass correlation coefficients were $\geq 0.60$ for most of the sperm parameters, indicating substantial reliability.

\section{Conclusion:}

The type and strength of associations between abstinence period and the quality of semen depend on underlying pathologies as well as on the specific parameters selected for investigation. Therefore, it is difficult to provide a clear recommendation for individual cases. If an improvement in concentration is the primary goal, prolonging the abstinence time to more than 7 days may be favourable; however, to prevent excessive oxidative damage by ROS, we do not advise extended abstinence periods. For motility a shorter abstinence time seems to be beneficial. A longer abstinence time is associated with a higher percentage of morphologically normal spermatozoids in men diagnosed with OAT. Overall, the collection strategy has to be based on the desired result, with longer abstinence time being positively associated with concentration, and shorter abstinence time related to better motility.

\section{Declarations}

\section{Ethics approval and consent to participate}

This study was approved by the local ethics committee (KEK-ZH-Nr. 2015-0534) and was carried out in accordance with relevant guidelines and regulations. Informed consent was obtained by all participants for this study.

\section{Consent for publication}

Consent for publication was obtained by all participants for this study. 
2 The data and material is available with request to the corresponding author.

\section{Competing interests}

$4 \quad$ Not applicable.

\section{$5 \quad$ Funding}

$6 \quad$ Not applicable.

\section{Authors contributions}

8 Min Xie: Investigator, concept of study, analysis of samples, management databank, finalization of the manuscript,

9 final approval of manuscript; Silvan Haemmerli: Concept of study, data analysis, interpretation of data, finalization

10 and final approval of manuscript; Kerstin Blickenstorfer: Investigator, participation in study design, analysis and

11 interpretation of data, finalization of the manuscript, final approval of manuscript; Brigitte Leeners: Principal investigator, concept and conduct of study, collection and analysis of data, preparation and finalization of manuscript, final approval of manuscript.

\section{Acknowledgements}

Not applicable.

(8)

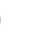

0


TABLES

2

3

Table 1: Sperm quality categories

\begin{tabular}{|l|l|}
\hline Normal samples & $\begin{array}{l}\geq 15 \text { million spermatozoa per ml and } \geq 32 \% \text { progressively motile spermatozoa and } \\
\geq 4 \% \text { spermatozoa with normal forms }\end{array}$ \\
\hline Oligozoospermia & $<15$ million spermatozoa per $\mathrm{ml}$ \\
\hline Asthenozoospermia & $<32 \%$ progressively motile spermatozoa \\
\hline Teratozoospermia & $<4 \%$ spermatozoa with normal morphology \\
\hline Oligoasthenoteratozoospermia & $\begin{array}{l}\text { spermatozoa }<15 \mathrm{~m} / \mathrm{ml} \text { and }<32 \% \text { progressive motile spermatozoa and } \\
<4 \% \text { spermatozoa with normal forms }\end{array}$ \\
\hline
\end{tabular}

Categories according to WHO lower reference limits

11 Table 2: Semen parameters

\begin{tabular}{|c|c|c|c|c|c|c|c|c|c|c|}
\hline & \multicolumn{2}{|c|}{ Normal samples } & \multicolumn{2}{|c|}{$\begin{array}{l}\text { Oligozoospermic } \\
\text { samples }\end{array}$} & \multicolumn{2}{|c|}{$\begin{array}{l}\text { Asthenozoospermic } \\
\text { samples }\end{array}$} & \multicolumn{2}{|c|}{$\begin{array}{l}\text { Teratozoospermic } \\
\text { samples }\end{array}$} & \multicolumn{2}{|c|}{ OAT samples } \\
\hline & $\mathrm{N}$ & Mean \pm SD & $\mathrm{N}$ & Mean \pm SD & $\mathrm{N}$ & Mean \pm SD & $\mathrm{N}$ & Mean \pm SD & $\mathrm{N}$ & Mean \pm SD \\
\hline $\begin{array}{l}\text { Sperm concentration } \\
(\mathrm{m} / \mathrm{ml})\end{array}$ & 1256 & $62.78 \pm 43.37$ & 1769 & $3.72 \pm 4.43$ & 1714 & $24.76 \pm 28.33$ & 998 & $24.29 \pm 26.17$ & 405 & $5.44 \pm 4.30$ \\
\hline Ejacualte volume (ml) & 1252 & $3.51 \pm 1.62$ & 1748 & $3.42 \pm 1.72$ & 1705 & $3.31 \pm 1.67$ & 994 & $3.40 \pm 1.63$ & 404 & $3.51 \pm 1.69$ \\
\hline $\begin{array}{l}\text { Progressively motile } \\
\text { spermatozoa (\%) }\end{array}$ & 1256 & $49.55 \pm 11.69$ & 1164 & $23.72 \pm 17.33$ & 1714 & $17.64 \pm 9.08$ & 990 & $27.78 \pm 17.33$ & 405 & $15.10 \pm 8.80$ \\
\hline $\begin{array}{l}\text { Total motile spermatozoa } \\
(\%)\end{array}$ & 1256 & $61.92 \pm 11.97$ & 1174 & $35.51 \pm 20.51$ & 1714 & $32.69 \pm 15.61$ & 991 & $43.09 \pm 19.50$ & 405 & $28.27 \pm 14.17$ \\
\hline TMSC $^{1}$ & 1252 & $128.50 \pm 99.70$ & 1132 & $6.18 \pm 8.24$ & 1680 & $16.44 \pm 24.85$ & 987 & $41.53 \pm 58.79$ & 403 & $3.45 \pm 4.54$ \\
\hline $\begin{array}{l}\text { Spermatozoa with } \\
\text { normal morphology }(\%)\end{array}$ & 1256 & $14.28 \pm 8.85$ & 876 & $4.94 \pm 5.63$ & 1324 & $6.47 \pm 6.82$ & 998 & $1.35 \pm 1.10$ & 405 & $1.30 \pm 1.29$ \\
\hline
\end{tabular}

13 
1 Table 3: Association between abstinence time and various factors of different sperm pathologies

\begin{tabular}{|l|c|c|c|}
\hline & Beta & SE & P-value \\
\hline Independent variables & \multicolumn{2}{|l|}{} \\
\hline All samples (n=4303) & & & \\
\hline Sperm concentration (m/ml) & 0.149 & 0.001 & $<\mathbf{0 0 1}$ \\
Progressively motile spermatozoa (\%) & -0.084 & 0.003 & $<\mathbf{0 . 0 0 1}$ \\
Spermatozoa with normal forms (\%) & -0.033 & 0.005 & $\mathbf{0 . 0 3 0}$ \\
\hline Normal samples (n=2381) & & & \\
Sperm concentration (m/ml) & 0.162 & 0.001 & $<\mathbf{0 . 0 0 1}$ \\
Progressively motile spermatozoa (\%) & -0.057 & 0.004 & $\mathbf{0 . 0 0 5}$ \\
Spermatozoa with normal forms (\%) & -0.043 & 0.007 & $\mathbf{0 . 0 3 9}$ \\
\hline Oligozoospermia (n=888) & & & \\
Sperm concentration (m/ml) & 0.052 & 0.032 & 0.125 \\
Progressively motile spermatozoa (\%) & -0.049 & 0.007 & 0.147 \\
Spermatozoa with normal forms (\%) & -0.033 & 0.012 & 0.329 \\
\hline Asthenozoospermia (n=704) & & & \\
Sperm concentration (m/ml) & 0.130 & 0.003 & $\mathbf{0 . 0 0 1}$ \\
Progressively motile spermatozoa (\%) & -0.041 & 0.019 & 0.274 \\
Spermatozoa with normal forms (\%) & -0.016 & 0.010 & 0.661 \\
\hline Teratozoospermia (n=1077) & & & $<.0 .019$ \\
Sperm concentration (m/ml) & 0.206 & 0.003 & $<0.001$ \\
Progressively motile spermatozoa (\%) & -0.138 & 0.004 & $<\mathbf{0 . 0 1}$ \\
Spermatozoa with normal forms (\%) & 0.051 & 0.096 & 0.090 \\
\hline Oligoasthenoteratozoospermia (n=276) & -0.039 & 0.025 & 0.517 \\
Sperm concentration (m/ml) & 0.149 & 0.253 & $\mathbf{0 . 0 1 3}$ \\
\hline Progressively motile spermatozoa (\%) & & & \\
Spermatozoa with normal forms (\%) & & & \\
\hline
\end{tabular}

21 Multiple regression analysis with number of days as dependent variable

22

Table 4: Correlation between investigated semen parameters and abstinence period in normal semen samples

\begin{tabular}{|c|c|c|c|c|c|c|c|c|c|c|}
\hline \multirow{3}{*}{ Normal samples } & \multicolumn{6}{|c|}{ Abstinence time intervals } & \multirow{3}{*}{$\begin{array}{c}\text { Overall } \\
\text { significance: } \\
\text { p-value }\end{array}$} & \multicolumn{3}{|c|}{ p-values } \\
\hline & \multicolumn{2}{|c|}{$0-2$ days } & \multicolumn{2}{|c|}{ 3-7 days } & \multicolumn{2}{|c|}{$>7$ days } & & $0-2$ to $3-$ & $3-7$ to & $0-2$ to \\
\hline & $\mathrm{N}$ & median & $\mathrm{N}$ & median & $\mathrm{N}$ & median & & 7 days & $>7$ days & $>7$ days \\
\hline Sperm concentration $(\mathrm{m} / \mathrm{ml})$ & 25 & 50.0 & 1155 & 50.0 & 76 & 73.5 & $<0.001^{*}$ & 1.00 & $<0.001 *$ & 0.218 \\
\hline Volume (ml) & 25 & 1.7 & 1151 & 3.3 & 76 & 3.7 & $<0.001 *$ & $<0.001 *$ & $0.002 *$ & $<0.001 *$ \\
\hline Progressively motile spermatozoa (\%) & 25 & 51.0 & 1155 & 48.0 & 76 & 46.0 & 0.230 & & & \\
\hline TMSC & 25 & 56.8 & 1151 & 100.3 & 76 & 156.6 & $<0.001 *$ & $<0.001^{*}$ & $<0.001 *$ & $<0.001 *$ \\
\hline Total motile spermatozoa (\%) & 25 & 68.0 & 1155 & 62.0 & 76 & 59.0 & 0.087 & & & \\
\hline Spermatozoa with normal forms (\%) & 25 & 10.0 & 1155 & 12.0 & 76 & 12.0 & 0.926 & & & \\
\hline
\end{tabular}

* statistically significant 
1

2

3

4

a) Normal samples

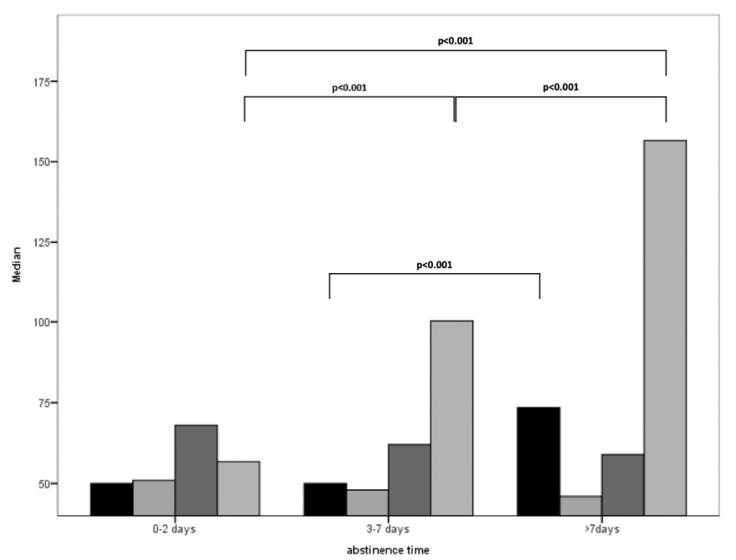

5

6

7

8

c) Asthenozoospermic samples

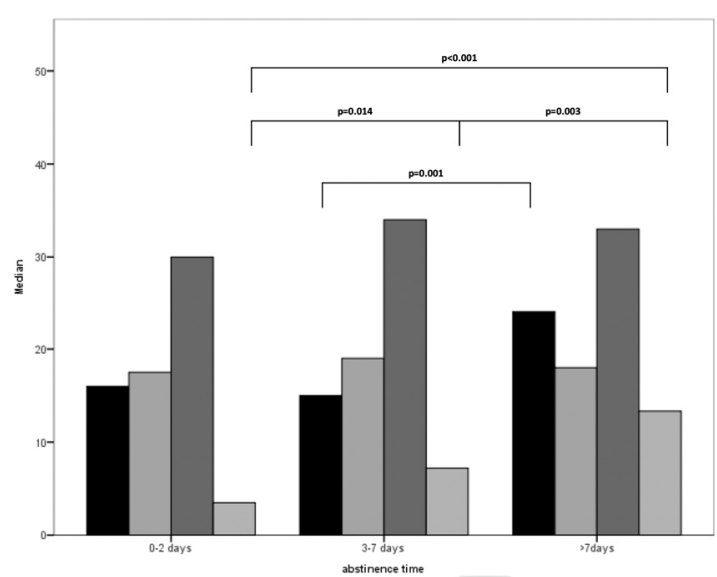

e) Oligoasthenoteratozoospermic samples

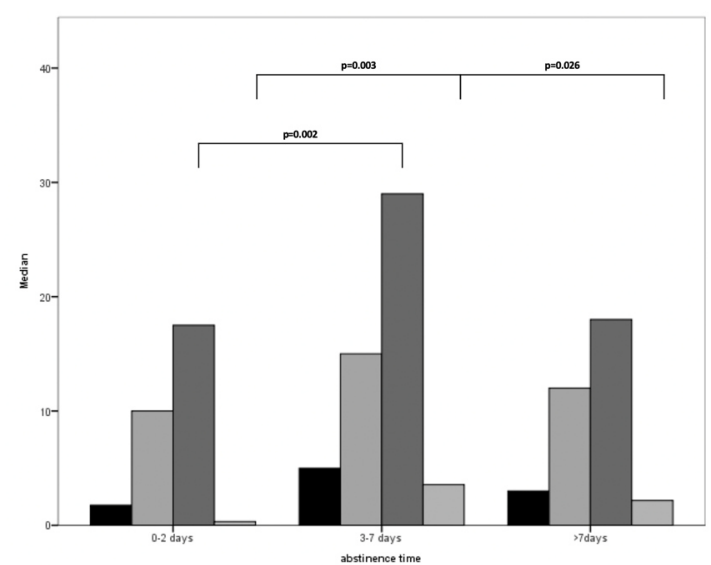

b) Oligozoospermic samples

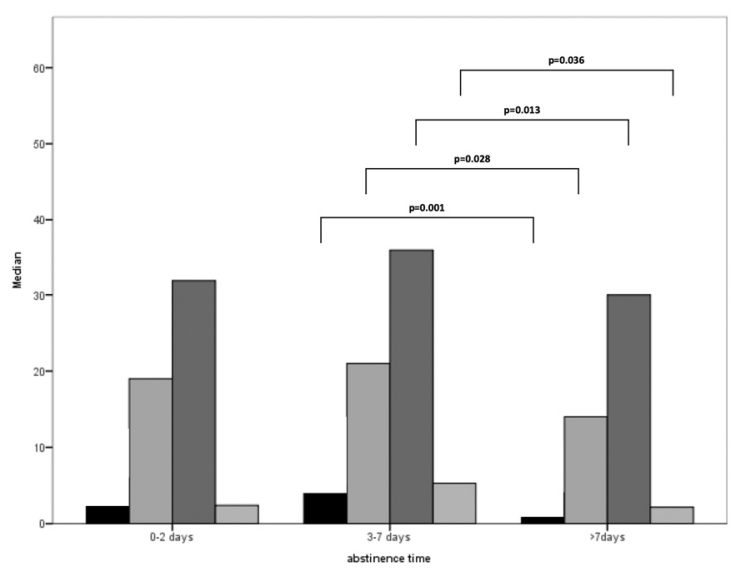

d) Teratozoospermic samples

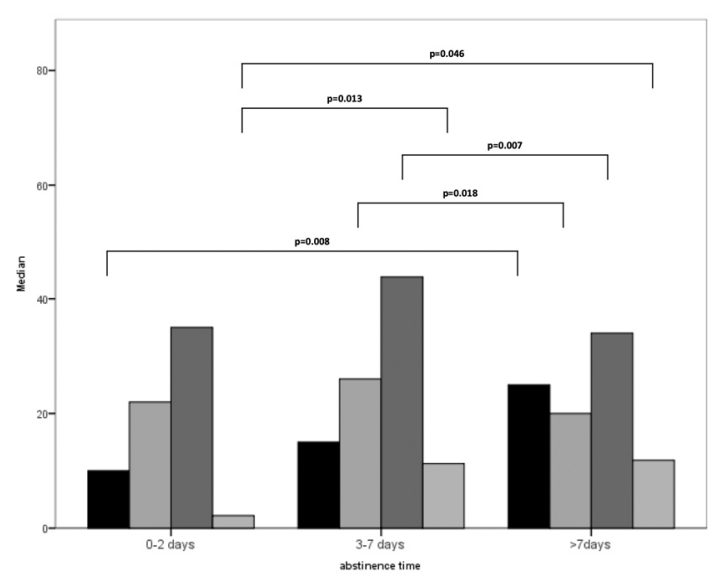

Legend to Figure 1a-e:

sperm concentration (Mio/ml)

$\square$ progressively motile spermatozoa (\%)

$\square$ total motile spermatozoa (\%)

total motile sperm count

Adjusted p-values are presented. 


\section{REFERENCES}

[1] Brugh VM, Lipshultz LI. Male factor infertility: evaluation and management. Medical Clinics. 2004;88: 36785.

[2] Hirsh A. Male subfertility. Bmj. 2003;327: 669-72.

[3] Donnelly ET, Lewis SE, McNally JA, Thompson W. In vitro fertilization and pregnancy rates: the influence of sperm motility and morphology on IVF outcome. Fertility and sterility. 1998;70: 305-14.

[4] Lemmens L, Kos S, Beijer C, et al. Predictive value of sperm morphology and progressively motile sperm count for pregnancy outcomes in intrauterine insemination. Fertility and sterility. 2016;105: 1462-68.

[5] Ombelet W, Dhont N, Thijssen A, Bosmans E, Kruger T. Semen quality and prediction of IUI success in male subfertility: a systematic review. Reproductive biomedicine online. 2014;28: 300-09.

[6] Lehavi O, Botchan A, Paz G, et al. Twenty-four hours abstinence and the quality of sperm parameters. Andrologia. 2014;46: 692-97.

[7] Tur-Kaspa I, Maor Y, Levran D, Yonish M, Mashiach S, Dor J. How often should infertile men have intercourse to achieve conception? Fertility and sterility. 1994;62: 370-75.

[8] Makkar G, Ng E, Yeung W, Ho P-C. A comparative study of raw and prepared semen samples from two consecutive days. The Journal of reproductive medicine. 2001;46: 565-72.

[9] Deveneau NE, Sinno O, Krause M, et al. Impact of sperm morphology on the likelihood of pregnancy after intrauterine insemination. Fertility and sterility. 2014;102: 1584-90. e2.

[10] van den Hoven L, Hendriks JC, Verbeet JG, Westphal JR, Wetzels AM. Status of sperm morphology assessment: an evaluation of methodology and clinical value. Fertility and sterility. 2015;103: 53-58.

[11] Høst E, Lindenberg S, Ernst E, Christensen F. Sperm morphology and IVF, Embryo quality in relation to sperm morphology following the WHO and Krüger's strict criteria. Acta obstetricia et gynecologica Scandinavica. 1999;78: 526-29.

[12] Organization WH. WHO Laboratory Manual for the Examination and Processing of Human Semen. Geneva, Switzerland: World Health Organization; 2010. 2020.

[13] Hornstein MD, Cohen JN, Thomas PP, Gleason RE, Friedman AJ, Mutter GL. The effect of consecutive day inseminations on semen characteristics in an intrauterine insemination program. Fertility and sterility. 1992;58: 433-35.

[14] Matilsky M, Battino S, Ben-Ami M, Geslevich Y, Eyali V, Shalev E. The effect of ejaculatory frequency on semen characteristics of normozoospermic and oligozoospermic men from an infertile population. Human Reproduction. 1993;8: 71-73.

[15] Levitas E, Lunenfeld E, Weiss N, et al. Relationship between the duration of sexual abstinence and semen quality: analysis of 9,489 semen samples. Fertility and sterility. 2005;83: 1680-86.

[16] Elzanaty S, Malm J, Giwercman A. Duration of sexual abstinence: epididymal and accessory sex gland secretions and their relationship to sperm motility. Human Reproduction. 2005;20: 221-25.

[17] Magnus Ø, Tollefsrud A, Árbyholm T, Purvis K. Effects of varying the abstinence period in the same individuals on sperm quality. Archives of andrology. 1991;26: 199-203.

[18] Agarwal A, Gupta S, Du Plessis S, et al. Abstinence time and its impact on basic and advanced semen parameters. Urology. 2016;94: 102-10.

[19] Gunn DD, Bates GW. Evidence-based approach to unexplained infertility: a systematic review. Fertility and sterility. 2016;105: 1566-74. e1.

[20] Menkveld R, Kruger T. Advantages of strict (Tygerberg) criteria for evaluation of sperm morphology. International journal of andrology. 1995;18: 36-42.

[21] Carlsen E, Petersen JH, Andersson A-M, Skakkebaek NE. Effects of ejaculatory frequency and season on variations in semen quality. Fertility and sterility. 2004;82: 358-66.

[22] De Jonge C, LaFromboise M, Bosmans E, Ombelet W, Cox A, Nijs M. Influence of the abstinence period on human sperm quality. Fertility and sterility. 2004;82: 57-65.

[23] Johnson L, Varner DD. Effect of daily spermatozoan production but not age on transit time of spermatozoa through the human epididymis. Biology of reproduction. 1988;39: 812-17.

[24] Blackwell JM, Zaneveld LJ. Effect of abstinence on sperm acrosin, hypoosmotic swelling, and other semen variables. Fertility and sterility. 1992;58: 798-802.

[25] Marshburn PB, Giddings A, Causby S, et al. Influence of ejaculatory abstinence on seminal total antioxidant capacity and sperm membrane lipid peroxidation. Fertility and sterility. 2014;102: 705-10.

[26] De Lamirande E, Gagnon C. Impact of reactive oxygen species on spermatozoa: a balancing act between beneficial and detrimental effects. Human Reproduction. 1995;10: 15-21.

[27] Sharma RK, Sabanegh E, Mahfouz R, Gupta S, Thiyagarajan A, Agarwal A. TUNEL as a test for sperm DNA damage in the evaluation of male infertility. Urology. 2010;76: 1380-86.

[28] Sikka SC. Relative impact of oxidative stress on male reproductive function. Current medicinal chemistry. 2001;8: 851-62.

[29] Elzanaty S, Richthoff J, Malm J, Giwercman A. The impact of epididymal and accessory sex gland function on sperm motility. Human Reproduction. 2002;17: 2904-11. 
1 [30] Fourie M, Toit DD, Bornman M, Van Der Merwe M-P, Du Plessis D. $\alpha$-Glucosidase, sperm ATP concentrations, and epididymal function. Archives of andrology. 1991;26: 139-41.

[31] de Jong DC, Reis HT. Sexual kindred spirits: Actual and overperceived similarity, complementarity, and partner accuracy in heterosexual couples. Personality and Social Psychology Bulletin. 2014;40: 1316-29.

[32] Mayorga-Torres BJM, Camargo M, Agarwal A, Du Plessis SS, Cadavid ÁP, Maya WDC. Influence of ejaculation frequency on seminal parameters. Reproductive Biology and Endocrinology. 2015;13: 1-7.

[33] Aziz N, Saleh RA, Sharma RK, et al. Novel association between sperm reactive oxygen species production, sperm morphological defects, and the sperm deformity index. Fertility and sterility. 2004;81: 349-54.

[34] Alvarez C, Castilla J, Martinez L, Ramirez J, Vergara F, Gaforio J. Biological variation of seminal parameters in healthy subjects. Human Reproduction. 2003;18: 2082-88.

[35] Keel BA. Within-and between-subject variation in semen parameters in infertile men and normal semen donors. Fertility and sterility. 2006;85: 128-34.

[36] Leushuis E, Van Der Steeg JW, Steures P, et al. Reproducibility and reliability of repeated semen analyses in male partners of subfertile couples. Fertility and sterility. 2010;94: 2631-35. 